\title{
Original Research \\ Evaluation of the clinical significance of serum pentraxin-3 levels in patients with polycystic ovary syndrome
}

\author{
Fatih Yeşi1 ${ }^{1}$, Köksal Deveci ${ }^{1}$, Pervin $\mathrm{Karli}^{2, *}$ \\ ${ }^{1}$ Department of Biochemistry, Gaziosmanpaşa University, Faculty of Medicine, 60250 Tokat, Turkey \\ ${ }^{2}$ Department of Obstetrics and Gynecology, İstinye University Medical Park Hospital, 34250 Samsun, Turkey \\ *Correspondence: parpi2300@hotmail.com (Pervin Karli) \\ Academic Editor: Michael H. Dahan \\ Submitted: 7 February 2021 Revised: 11 April 2021 Accepted: 22 April 2021 Published: 9 February 2022
}

\begin{abstract}
Background: Polycystic ovary syndrome (PCOS) is commonly associated with insulin resistance (IR), hyperinsulinemia, and dyslipidemia, which play a role in the development of endothelial dysfunction and promote the early onset of cardiovascular diseases. The aim of this study was to evaluate the clinical importance of pentraxin-3 levels in PCOS patients. Methods: Forty-five female patients diagnosed with PCOS according to the 2003 Rotterdam criteria and 42 healthy women were included in the study. All women studied were tested within 3 and 5 days of their menstrual cycle. Ultrasonographic evaluation of each patient was first conducted using the suprapubic method. Serum PTX-3, endothelin 1 (ET-1), vascular cell adhesion molecule 1 (VCAM-1), intercellular adhesion molecule 1 (ICAM-1), and nitric oxide levels were measured using the enzyme-linked immunosorbent assay method. Results: Pentraxine-3 (PTX-3) levels in the patient group were significantly lower than those in the control group $(p<0.05)$. PTX-3 levels in the group with hirsutism were significantly higher than those in the nonhirsutism group $(p<0.05)$. Homeostatic model assessment for IR (HOMA-IR) levels in the patient group were significantly higher than those in the control group $(p<0.01)$. A weak negative correlation was found between PTX-3 and HbA1c levels. The accuracy rate of the PTX-3 test in distinguishing patients and nonpatients was moderate with a 0.634 area-under-the-curve value. Conclusions: During the follow-up of patients with PCOS, a decrease in serum PTX-3 levels associated with hirsutism and IR may be observed.
\end{abstract}

Keywords: PCOS; Endothelium dysfunction; Pentraxin-3; HOMA-IR

\section{Introduction}

Polycystic ovary syndrome (PCOS) is the most common endocrine disorder among women, with an estimated prevalence of 4-12\% worldwide [1]. Women with PCOS have several cardiovascular risk factors, such as obesity, lipid abnormalities, impaired glucose tolerance, and hypertension. Recent data have shown an increased prevalence of cardiovascular disease (CVD) in women with PCOS [2,3]. Patients with PCOS are also at risk of developing CVD from an increase in hypertension and dyslipidemia [4].

When metabolic desorders (insulin resistance, hiperinslunemi, dyslipidemia) coexist with PCOS, they may cause the development of endothelial dysfuntion and early onset of CVD [5,6]. Various studies and metaanalyses have shown that PCOS patients have endothelial disfunction (ED), endothelial inflammation, endothelial cell proliferation, and associated coagulation disorders $[7,8]$. While the causes of endothelial dysfuntion (ED) in PCOS remain to be established, possibilities include cardiovascular risk factor, inflammatory markers or endocrine changes [8].

Pentraxine-3 (PTX-3) is an acute-phase reactant that forms the humoral part of the innate immune system and plays a key role as an effector and modulator in inflammation. PTX-3 is involved in vascular inflammation and endothelial dysfunction through a variety of mechanisms. Studies have confirmed an important link between PTX3 and endothelial dysfunction and have identified several pathogenetic pathways. PTX-3 stimulates vascular inflammation by modulating inflammatory cells. It also decreases nitric oxide (NO) synthesis in endothelial cells, inhibits cell proliferation and changes its functions. PTX-3 blocks the action of fibroblast growth factor 2 (FGF2) by inactivating these molecules and forming a molecular complex. By interacting with $\mathrm{P}$-selectin, it promotes the vascular inflammatory response and endothelial dysfunction. PTX-3 also increases the synthesis of matrix metalloproteinase either directly or by blocking NO synthesis [3,9].

Altered expression of PTX-3 is also associated with increased inflammation in the ovary and stimulation of folliculogenesis [10]. In addition, PTX-3 is positively associated with arterial hypertension, flow-mediated expansion, and intima-media thickness; therefore, the role of PTX-3 in the pathogenesis and evaluation of endothelial dysfunction is well established and may serve as a biomarker for PCOS [11].

The aim of this study was to evaluate the clinical importance of pentraxin-3 levels in PCOS patients. 
Table 1. Demographic data for the patient and control groups.

\begin{tabular}{lccc}
\hline & Patient (n: 45) & Control (n: 42) & $p$ \\
\hline Age (years) & $22.11 \pm 6.16$ & $23.76 \pm 5.34$ & $>0.05$ \\
BMI & $25.37 \pm 6.21$ & $22.10 \pm 3.44$ & $<0.05^{* *}$ \\
Abdominal circumference (cm) & $76.88 \pm 13.67$ & $71.26 \pm 8.57$ & $<0.05^{* *}$ \\
Ultrasonographic subcutaneous fat thickness (mm) & $25.08 \pm 9.28$ & $20.23 \pm 6.64$ & $<0.05^{* *}$ \\
Smoking & $8(17.9 \%)$ & $11(25.6 \%)$ & $>0.05$ \\
Additional disease & $5(11.1 \%)$ & $6(9.5 \%)$ & $>0.05$ \\
Additional drug usage & $7(15.6 \%)$ & $2(4.8 \%)$ & $>0.05$ \\
Regular menstrual cycle & $4(8.9 \%)$ & $39(92.9 \%)$ & $<0.01^{*}$ \\
Hirsutism & $30(66.7 \%)$ & $3(7.1 \%)$ & $<0.01^{*}$ \\
Medication & $9(20 \%)$ & $0(0 \%)$ & $<0.05^{* *}$ \\
\hline
\end{tabular}

Notes: BMI, body mass index.

$* p<0.01, * * p<0.05$.

\section{Materials and methods}

This cross-sectional study involved 45 women (study group) diagnosed with PCOS using the Rotterdam 2003 consensus by experts from the American Society for Reproductive Medicine (ASRM) and the European Society for Human Reproduction and Embryology (ESHRE) (Rotterdam criteria 4.8), along with 42 healthy persons (control group). Those who met the inclusion criteria were provided informed consent to participate in the study. The exclusion criteria were as follows: (1) pregnancy; (2) any endocrine disorder, such as Cushing's syndrome; (3) 21hydroxylase deficiency; (4) congenital adrenal hyperplasia; (5) history of diabetes and gestational diabetes or (6) chronic maladies, such as CVD, hepatic disease, hematological disease, chronic renal failure, or cancer. The study comprised adult volunteers who applied to the obstetrics and gynecology outpatient clinic of the Amasya Şerafeddin Sabuncuoğlu Training and Research Hospital in Turkey with symptoms of PCOS and where PCOS was diagnosed. The control group comprised women who applied to the same hospital with problems that required counseling, but for whom PCOS was not diagnosed after clinical examination. The aim and methods of the study were verbally explained to all volunteers in the control group, who had sociodemographic characteristics similar to those of the patient group. All volunteers gave written informed consent. The study was conducted with the permission of the clinical research ethics committee of Tokat Gaziosmanpaşa University (Decision no: 83116987-014).

All of the women studied were tested within 3-5 days of their menstrual cycle. Anthropometric measurements (body mass and height) were taken, and the body mass index (BMI) was calculated according to the standard formula using the bioimpedance method with Bodystat 1500 (Bodystat, Douglas, Isle of Man). Fifteen-milliliter samples of venous blood were withdrawn between 8:00 and 9:00 AM after a 16 -h overnight fast. Serum and plasma samples were stored at $-80^{\circ} \mathrm{C}$. The blood samples were collected accord- ing to the manufacturer's recommendations.

\subsection{Biochemical analysis}

Routine biochemical analyses of the blood samples were conducted using the Roche Cobas 8000 (Roche, Basel, Switzerland) while hormone analyses were conducted using Siemens Advia Centaur XP (Siemens, Munich, Germany). PTX-3, endothelin 1 (ET-1), vascular cell adhesion molecule 1 (VCAM-1), intercellular adhesion molecule 1 (ICAM-1), and NO concentrations were measured using the enzyme-linked immunosorbent assay (ELISA) method and presented as ng/mL. Commercially available human PTX3, ET-1, VCAM-1, ICAM-1, and NO ELISA kits (Bioassay Technology Laboratory, Shangai, China) were used. Kits' sensitivity are $1.01 \mathrm{ng} / \mathrm{L}$ and $\mathrm{CV}(\%)=\mathrm{SD} /$ mean $\times 100$ intra-assay: $\mathrm{CV}<8 \%$ inter assay $<10 \%$. Ultrasonographic evaluation of each patient was first conducted using the suprapubic method.

\subsection{Statistical analyses}

Statistical analyses of the data were conducted using SPSS 25 (IBM Corp., Armonk, NY, USA). In the analyses, the routine laboratory data on the participants, their hormonal levels, and their normal distribution of PTX-3, NO, VCAM-1, ICAM-1, and ET-1 were evaluated using the Kruskal-Wallis analysis of variance. The parameters conforming to the normal distributions were given as the mean $\pm \mathrm{SD}$; those that did not conform were given as the median (min-max). The Student's $T$-test was used for a paired comparison of parameters that conformed to normal distribution; the Mann-Whitney U test was used for a paired comparison of parameters that did not comply with the normal distribution. The relationship between noncategorical variables was analyzed using Spearman's rho correlation analysis, and the relationship between categorical variables was examined using the chi-squared test. The analysis of the receiver operating characteristic curve (ROC) was used to determine the diagnostic competence of PTX-3. 
Table 2. Routine laboratory data on the patient and control groups.

\begin{tabular}{lccc}
\hline & Patient (n: 45) & Control (n: 42) & $p$ \\
\hline Glucose (mg/dL) & $89.4 \pm 8.7$ & $88.6 \pm 9.4$ & $>0.05$ \\
Triglycerides (mg/dL) & $81.0(40.0-431.0)$ & $79.0(34.0-227.0)$ & $>0.05$ \\
AST (U/L) & $20.17 \pm 7.4$ & $19.35 \pm 4.9$ & $>0.05$ \\
ALT (U/L) & $18.5 \pm 6.2$ & $14.7 \pm 6.1$ & $>0.05$ \\
BUN (mg/dL) & $22.15 \pm 5.5$ & $21.7 \pm 5.95$ & $>0.05$ \\
Creatinine (mg/dL) & $0.65 \pm 0.09$ & $0.66 \pm 0.07$ & $>0.05$ \\
HbA1c (mg/dL) & $5.40 \pm 0.22$ & $5.41 \pm 0.43$ & $>0.05$ \\
FSH (U/L) & $5.19(0.40-20.20)$ & $5.89(2.26-10.36)$ & $>0.05$ \\
LH (U/L) & $7.87(5.65-29.07)$ & $5.94(0.53-33.96)$ & $<0.05 * *$ \\
LH/FSH (U/L) & $1.516(0.6-4.27)$ & $1.008(0.02-7.19)$ & $<0.05 * *$ \\
Estradiol (ng/L) & $103.8(11.8-237.6)$ & $88.2(26.5-332.8)$ & $>0.05$ \\
Progesterone (ug/L) & $0.95(0.21-11.75)$ & $1.06(0.21-21.35)$ & $>0.05$ \\
17-OH Progesterone (pg/mL) & $1.5(0.2-3.2)$ & $1.6(0.3-3.1)$ & $>0.05$ \\
Prolactin (ug/L) & $13.91(6.12-31.71)$ & $12.38(4.71-24.47)$ & $>0.05$ \\
DHEAS (ug/L) & $257.0(58.04-549.7)$ & $258.0(113.3-650.4)$ & $>0.05$ \\
Testosterone (ng/dL) & $36.27(21.88-55.24)$ & $33.44(14.08-58.55)$ & $>0.05$ \\
Free T4 (ng/dL) & $1.27(0.91-1.54)$ & $1.23(0.92-3.58)$ & $>0.05$ \\
TSH (mU/L) & $2.09(0.13-10.33)$ & $1.94(0.88-3.47)$ & $>0.05$ \\
Insulin (mU/L) & $0.71(0.23-11.75)$ & $0.92(0.27-14.47)$ & $>0.05$ \\
HOMA-IR (mU/L) & $2.55(0.56-4.99)$ & $2.11(0.55-37.44)$ & $<0.01^{*}$ \\
\hline Notes: AST, aspartan & & & \\
& & &
\end{tabular}

Notes: AST, aspartate aminotransferase; ALT, alanine aminotransferase; BUN, blood urea nitrogen; WBC, white blood cell; HbA1c, hemoglobin A1c; FSH, follicle stimulating hormone; LH, luteinizing hormone; DHEAS, dehydroepiandrosterone sulfate; TSH, thyroid stimulating hormone.

$* p<0.01, * * p<0.05$.

\section{Results}

Body mass index, abdominal circumference and subcutaneous thickness were found statistically significant in the study group. Also, irregular menstrual cycles and hirsutism levels were higher in the study group $(p<0.05)$ (Table 1).

When comparing hormonal measurement levels, we observed that only the levels of luteinizing hormone (LH) in the participants were statistically significantly different $(p<0.05)$. That is, no significant difference was observed in the other hormone and biochemical measurement levels in both the patient and control groups (Table 2).

The value of the homeostatic model assessment for IR (HOMA-IR) of the patient group was statistically significantly higher than that in the control group $(p<0.01)$ (Table 2).

The PTX-3 levels in the patient group decreased compared to those in the control group (Fig. 1) and the difference was statistically significant $(p=0.032)$. NO $(p=$ $0.339)$, VCAM-1 $(p=0.363)$ ICAM-1 $(p=0.366)$, and endothelin-1 $(p=0.113)$ levels were not statistically significantly different (Table 3 ).

When the study parameters were compared according to the presence of hirsutism in the PCOS patient group, we

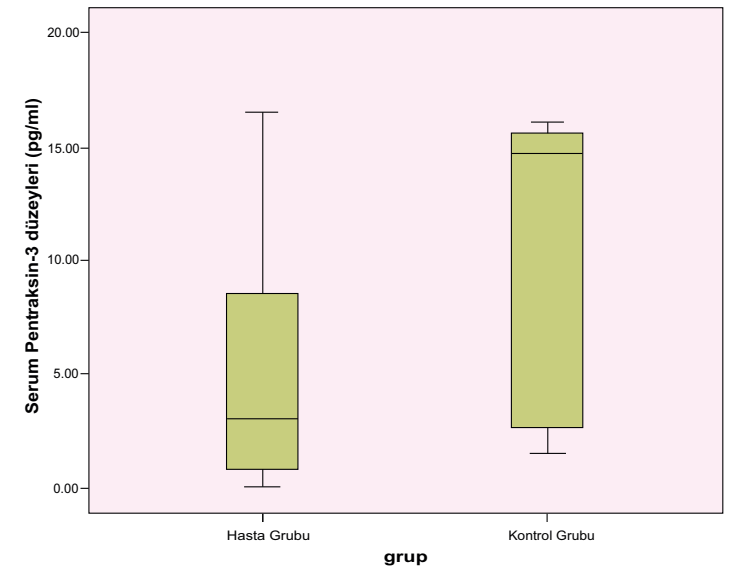

Fig. 1. Serum pentraxin-3 levels in the patient and control groups.

observed a statistically significant difference in the PTX$3(p=0.032)$ levels in the participants, while there was no statistically significant difference in the levels of $\mathrm{NO}$, VCAM-1, ICAM-1, endothelin-1, FSH, LH, progesterone, 17-OH progesterone, prolactin, DHEAS, and testosterone (Table 4). 
Table 3. PTX-3, NO, VCAM-1, ICAM-1, and ET values in the patient and control groups.

\begin{tabular}{lccc}
\hline & Patient (n: 45), median (min-max) & Control (n: 42), median (min-max) & $p$ \\
\hline PTX-3 $(\mathrm{ng} / \mathrm{mL})$ & $3.0(0.61-16.50)$ & $14.94(1.49-15.7)$ & 0.032 \\
NO $(\mu \mathrm{mol} / \mathrm{L})$ & $150.81(23.90-330.39)$ & $130.86(23.9-370.78)$ & 0.339 \\
VCAM-1 $(\mathrm{ng} / \mathrm{mL})$ & $16.03(1.48-32.53)$ & $14.31(3.02-31.72)$ & 0.363 \\
ICAM-1 $(\mathrm{ng} / \mathrm{L})$ & $2510.93(50.0-5862)$ & $2224.71(4-5834)$ & 0.366 \\
ET-1 $(\mathrm{ng} / \mathrm{L})$ & $204.06(4.0-452.46)$ & $159.08(2.46-440.2)$ & 0.113 \\
\hline
\end{tabular}

Notes: PTX-3, pentraxin-3; NO, nitric oxide; VCAM-1, vascular cell adhesion molecule-1; ICAM-1, intercellular adhesion molecule-1; ET-1, endothelin-1.

Table 4. Study parameters based on the presence of hirsutism in the patient group.

\begin{tabular}{lccc}
\hline & Hirsutism present (n: 30) & Hirsutism absent (n: 15) & $p$ \\
\hline PTX-3 (ng/mL) & $8.39(0.61-16.50)$ & $4.20(0.61-16.27)$ & 0.031 \\
NO $(\mu \mathrm{mol} / \mathrm{L})$ & $95.97(23.90-330.39)$ & $173.89(23.9-330.39)$ & 0.763 \\
VCAM-1 $(\mathrm{ng} / \mathrm{mL})$ & $14.75(1.48-32.53)$ & $21.24(3.02-31.72)$ & 0.455 \\
ICAM-1 $(\mathrm{ng} / \mathrm{L})$ & $2388(50-5790)$ & $2912(58-5862)$ & 0.500 \\
ET-1 $(\mathrm{ng} / \mathrm{L})$ & $174.2(10.5-452.46)$ & $131.2(4.0-440.2)$ & 0.971 \\
FSH $(\mathrm{U} / \mathrm{L})$ & $3.22(0.4-20.20)$ & $5.53(2.39-8.56)$ & 0.134 \\
LH $(\mathrm{U} / \mathrm{L})$ & $5.46(5.65-29.07)$ & $9.37(5.6-29.07)$ & 0.056 \\
Progesterone (ug/L) & $0.7(0.21-7.81))$ & $0.85(0.38-11.75)$ & 0.208 \\
17-OH Progesterone $(\mathrm{pg} / \mathrm{mL})$ & $1.6(0.7-3.0)$ & $1.3(0.2-3.2)$ & 0.571 \\
Prolactin (ug/L) & $14.21(6.12-31.71)$ & $13.4(6.71-18.11)$ & 0.379 \\
DHEAS (ug/L) & $266.75(163.0-549.7)$ & $240.0(58.04-427.0)$ & 0.151 \\
Testosterone (ng/dL) & $36.51(21.88-54.21)$ & $34.85(26.11-55.24)$ & 0.847 \\
\hline Notes: PTX-3, pentraxin-3; NO, nitric oxide; VCAM-1, vascular cell adhesion molecule-1; \\
ICAM-1, intercellular adhesion molecule-1; ET-1, endothelin-1; FSH, follicle stimulating hor- \\
mone; LH, luteinizing hormone; DHEAS, dehydroepiandrosterone sulfate. &
\end{tabular}

There was no statistically significant difference between the levels of PTX-3 and VCAM-1, NO, ICAM-1, ET-1 between the patient group and the control group $(p>$ $0.05)$, and there was a statistically significant relationship with glucose $(p<0.05)$ (Table 5).

Table 5. Relationship between pentraxin-3 levels and study parameters in the patient group.

\begin{tabular}{lcc}
\hline \multicolumn{3}{c}{ Pentraxin-3 } \\
\hline & R value & $p$ value \\
\hline VCAM-1 $(\mathrm{ng} / \mathrm{mL})$ & 0.262 & 0.083 \\
NO $(\mu \mathrm{mol} / \mathrm{L})$ & 0.267 & 0.076 \\
ICAM-1 $(\mathrm{ng} / \mathrm{L})$ & 0.276 & 0.066 \\
ET-1 $(\mathrm{ng} / \mathrm{L})$ & 0.188 & 0.217 \\
Glucose $(\mathrm{mg} / \mathrm{dL})$ & -0.304 & 0.042 \\
\hline
\end{tabular}

The accuracy rate of the PTX-3 test in distinguishing patients and nonpatients was moderate, with a 0.634 area-under-the-curve (AUC) value (Fig. 2). The confidence interval was narrow and the $\mathrm{p}$ value was significant $(p=$ 0.031 ). There was a $60.0 \%$ sensitivity and $64.3 \%$ specificity at 5.76 cutoff values; PCOS had a diagnostic capabil- ity with a sensitivity of $51.1 \%$ and specificity of $81.4 \%$ at a cutoff value of 8.34 (Table 6).

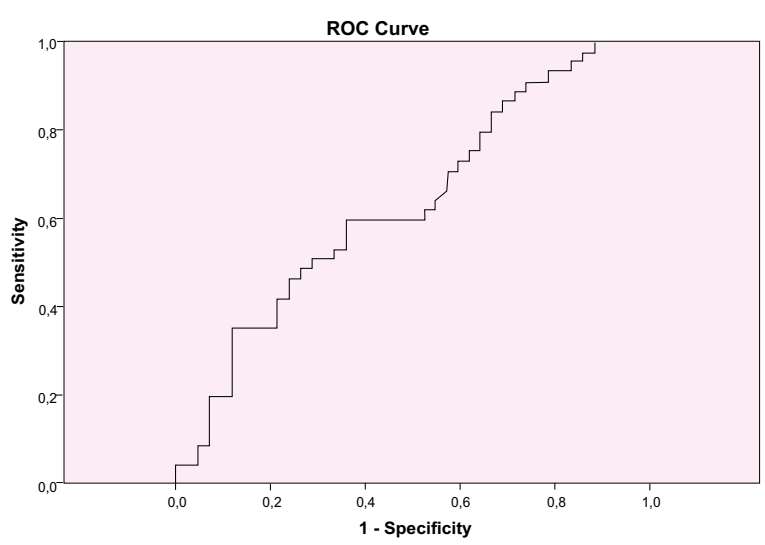

Fig. 2. Receiver operating characteristic (ROC) curve used to determine the diagnostic competence of pentraxin-3. 
Table 6. The Roc analysis in evaluation of the diagnostic adequacy of pentraxin-3.

\begin{tabular}{|c|c|c|c|c|c|c|c|}
\hline \multirow{2}{*}{ Area } & \multirow{2}{*}{ Standard error ${ }^{a}$} & \multirow{2}{*}{ Asymptotic Sig. ${ }^{b}$} & \multicolumn{2}{|c|}{ Asymptotic $95 \%$ Confidence Interval } & \multirow[b]{2}{*}{ Cutoff } & \multirow[b]{2}{*}{ Sensitivity } & \multirow[b]{2}{*}{ 1-specificity } \\
\hline & & & Lower bound & Upper bound & & & \\
\hline 0.634 & 0.060 & 0.031 & 0.517 & 0.751 & 5.76 & 0.60 & 0.357 \\
\hline Cutoff & Sensitivity & 1-specificity & & & 8.34 & 0.511 & 0.286 \\
\hline
\end{tabular}

a. Under the nonparametric assumption.

b. Null hypothesis:true area $=0.5$.

\section{Discussion}

In response to local inflammation and proinflammatory signals, PTX-3 is released from peripheral tissues; therefore, PTX-3 is not affected by drug-induced hepatic protein synthesis, making it a useful marker for inflammation [12]. In some studies, PTX-3 levels were examined in PCOS patients and were reported to be higher, lower, or similar to that in the control group. In addition, studies have shown that BMI and IR are either positively or negatively correlated with PTX-3 [13,14]. Obesity, IR and and PTX-3 play important role in PCOS etiology. In addition, PTX-3 deficiency is associated with severe impaired fertility [15]; however, it is unclear why PTX-3 levels in PCOS patients increase or decrease.

According to the literature the rate of obesity in women with PCOS varies between $30 \%$ and $75 \%$, with these individuals having especially abdominal obesity. Abdominal fat is accompanied by IR, increased BMI, and increased lipid profile and blood pressure [16,17]. IR plays either a direct or indirect role in PCOS [17]. In the present study, based on the World Health Organization's BMI classification, we observed that BMI, abdominal circumference, and the thickness of ultrasonographic subcutaneous fat in women with PCOS were higher than that in the control group, in line with data from other studies [16,17]; however, we could not determine relationship between BMI and PTX-3 levels. The incidence of menstrual dysfunction and hirsutism in PCOS patients was also higher than that in the control group.

In the pathogenesis of PCOS, the disease is believed to be the result of an irregular regulation between FSH and LH. The LH/FSH ratio is used as a diagnostic value for PCOS and is usually $1 / 1$. A high LH may be a possible indicator of PCOS. In the present study, the LH levels and $\mathrm{LH} / \mathrm{FSH}$ ratios in the patient group were statistically significantly higher than those in the control group. Although we observed that these results were comparable to those in the literature $[17,18]$, we also determined that the LH/FSH ratio we obtained was a diagnostic indicator or PCOS; however, we could not determine the relationship between LH levels or the LH/FSH ratio and PTX-3 in PCOS.

Aydoğdu et al. [19] reported that plasma PTX-3 and HOMA-IR are higher in women with PCOS than in healthy controls, and blood PTX-3 levels are positively correlated with BMI, waist-to-hip ratio (WHR), and HOMA-IR. Şahin et al. [12] have reported that PTX-3 levels in young women with PCOS are lower than those in the healthy control group, that their HOMA-IR and LH levels are higher, and that PTX-3 is negatively correlated with HOMA-IR and BMI but not with LH. Sari et al. [13] reported that IR and PTX-3 did not differ between their PCOS and control groups. In the present study, an increase in HOMA-IR values in PCOS patients was detected. Our findings, which show this increase in IR, led us to believe that, akin to the results of other studies [20], PTX-3 and HOMA-IR are negatively correlated and that low PTX-3 levels can be evaluated together with IR in the etiology of PCOS.

Although the relationships between PCOS and cardiometabolic abnormalities have been well established, it is not entirely clear whether PCOS is associated with subclinical and clinical CVD independent of these CVD risk factors [21,22]. Numerous studies have reported that endothelial dysfunction is commonly associated with PCOS [5]. IR can also be a factor in endothelial dysfunction because it increases the synthesis of vasoconstrictive agents, which leads to an increase in atherosclerosis and impairment of the vasodilating effect of insulin that is observed in women with PCOS [23]; however, recent studies to clarify the relationship between PCOS and cardiovascular diseases have focused on the PTX-3 marker [24,25]. Inflammation poses a risk for endothelial dysfunction by inhibiting endothelial NO synthase activity and reducing the number of endothelial progenitor cells [26,27]. Endothelial dysfunction is considered a first step in the atherosclerotic process [28]. PTX-3 is produced within the inflammation zone and is associated with endothelial dysfunction [25]; however, the extent to which the circulating PTX-3 levels is associated with PCOS remains unclear.

Some studies have shown that PTX-3 increases with PCOS [13]. PTX-3 levels are low in individuals with PCOS in studies wherein the patient group was mostly overweight and obese. Wyskide et al. [29] have reported that the decrease in PTX-3 concentrations detected in obese individuals is impaired in PCOS by microinflammation, and have indicated a possible dysfunction in stromal adipose tissue and liver steatosis reflected by increased IR. In the present study, we observed that women with PCOS had lower PTX-3 levels than those in the control group and that these results were consistent with those of other studies that showed a decrease in PTX-3 levels [12,14]. In patients with 
PCOS, no significant change was found in the levels of NO, endothelin-1, sICAM-1, and SVCAM-1, the other biomarkers for endothelial dysfunction.

When considering the relationship between hirsutism and pentraxin-3, which indicate other clinical symptoms of PCOS, we determined that hirsutism in the patient group was statistically higher compared to the control group. PTX-3 levels were increased in patients with hirsutism in the patient group, while NO, VCAM-1, ICAM1, Endothelin-1, FSH, LH, progesterone, 17-OH Progesterone, prolactin, DHEAS and testosterone levels remained statistically unchanged. As for the relationship between pentraxin-3 levels and other study parameters in patients, we found that PTX-3 was negatively correlated to glucose and $\mathrm{HbA1c}$ levels.

PTX-3 can be effective in the inflammatory process and the etiology of PCOS through only IR. This may explain the combination of atherosclerotic disease during the course of PCOS. In addition, it is known that PTX-3 levels increase with age [20,23]. In many studies on the atherosclerotic process, PTX-3 levels of middle-aged and elderly patients have been investigated [20]. In studies conducted on PCOS, patients predominantly in their twenties were examined. The process of atherosclerosis and its complications increase with age. The reason for the low PTX-3 levels in our study may be that the patients were relatively young and newly diagnosed. As the disease progresses, PTX-3 releases may dramatically increase secondary to sustained IR, oxidative stress, and proinflammatory cytokines; accordingly, additional studies on PTX3 with long-term follow-up involving middle-aged and elderly PCOS patients should be conducted in more detail.

Roc analysis is used in clinical epidemiology to measure how accurately medical diagnostic tests can distinguish patients and nonpatients. Herein, PCOS had a diagnostic capability with a sensitivity of $51.1 \%$ and specificity of $81.4 \%$ at a cutoff value of 8.34 .

\section{Conclusions}

PTX-3 serum levels, which are low in PCOS patients, could be used in the clinical follow-up of patients with PCOS as they are negatively associated with IR and are found in high levels in patients with hirsutism.

\section{Author contributions}

PK performed data collection and manuscript correction. FY and $\mathrm{KD}$, manuscript writing- editing-review, project-development analysis and manuscript writing. All authors read and approved the final manuscript.

\section{Ethics approval and consent to participate}

Informed consent was obtained from all individual participants included in the study. All procedures performed in the current study were in accordance with the ethical standards of the institutional research committee of the Scientific Research Ethical Committee at the Gaziosmanpaşa University and with the Helsinki declaration and its later amendments or comparable ethical standards. The protocol was approved by the Ethics Committee of "83116987-014".

\section{Acknowledgment}

This project was not supported by any instution. We would like to express our gratitude to all those who helped us during the writing of this manuscript. Thanks to all the peer reviewers for their opinions and suggestions.

\section{Funding}

This research received no external funding.

\section{Conflict of interest}

The authors declare no conflict of interest.

\section{References}

[1] Franik S, Kremer JAM, Nelen WLDM, Farquhar C. Aromatase inhibitors for subfertile women with polycystic ovary syndrome. Cochrane Database of Systematic Reviews. 2014; 2: CD010287.

[2] Wild S, Pierpoint T, McKeigue P, Jacobs H. Cardiovascular disease in women with polycystic ovary syndrome at long-term follow-up: a retrospective cohort study. Clinical Endocrinology. 2000; 52: 595-600.

[3] Tarkun I, Arslan BC, Cantürk Z, Türemen E, Sahin T, Duman C. Endothelial dysfunction in young women with polycystic ovary syndrome: relationship with insulin resistance and lowgrade chronic inflammation. Journal of Clinical Endocrinology \& Metabolism. 2004; 89: 5592-5596.

[4] Norman RJ, Dewailly D, Legro RS, Hickey TE. Polycystic ovary syndrome. Lancet. 2007; 370: 685-697.

[5] Diamanti-Kandarakis E, Alexandraki K, Piperi C, Protogerou A, Katsikis I, Paterakis T, et al. Inflammatory and endothelial markers in women with polycystic ovary syndrome. European Journal of Clinical Investigation. 2006; 36: 691-697.

[6] Cho LW, Randeva HS, Atkin SL. Cardiometabolic aspects of polycystic ovarian syndrome. Vascular Health and Risk Management. 2007; 3: 55-63.

[7] Toulis KA, Goulis DG, Mintziori G, Kintiraki E, Eukarpidis E, Mouratoglou S, et al. Meta-analysis of cardiovascular disease risk markers in women with polycystic ovary syndrome. Human Reproduction Update. 2011; 17: 741-760.

[8] Dube R. Does endothelial dysfunction correlate with endocrinal abnormalities in patients with polycystic ovary syndrome? Avicenna Journal of Medicine. 2016; 6: 91-102.

[9] Zeybek S. Tekrarlayan gebelik kaybı olan vakalarda Pentraxin 3 ekspresyon profilinin belirlenmesi. Pamukkale University Faculty of Medicine Department of Medical Genetics 812015

[10] Camaioni A, Klinger FG, Campagnolo L, Salustri A. The influence of pentraxin 3 on the ovarian function and its impact on fertility. Frontiers in Immunology. 2018; 9: 2808.

[11] Zlibut A, Bocsan IC, Agoston-Coldea L. Pentraxin-3 and endothelial dysfunction. Advances in Clinical Chemistry. 2019; 91: 163-179.

[12] Sahin FK, Sahin SB, Balik G, Ural UM, Tekin YB, Cure MC, et $a l$. Does low pentraxin-3 levels associate with polycystic ovary 
syndrome and obesity? International Journal of Clinical and Experimental Medicine. 2014; 7: 3512-3519.

[13] Sari U, Kaygusuz I, Kafali H. Is pentraxin 3 a new cardiovascular risk marker in polycystic ovary syndrome? Gynecologic and Obstetric Investigation. 2014; 78: 173-178.

[14] Tosi F, Di Sarra D, Bonin C, Zambotti F, Dall'Alda M, Fiers $\mathrm{T}$, et al. Plasma levels of pentraxin-3, an inflammatory protein involved in fertility, are reduced in women with polycystic ovary syndrome. European Journal of Endocrinology. 2014; 170: 401409.

[15] Salustri A, Garlanda C, Hirsch E, De Acetis M, Maccagno A, Bottazzi B, et al. PTX3 plays a key role in the organization of the cumulus oophorus extracellular matrix and in in vivo fertilization. Development. 2004; 131: 1577-1586.

[16] Moran LJ, Noakes M, Clifton PM, Wittert GA, Williams G, Norman RJ. Short-term meal replacements followed by dietary macronutrient restriction enhance weight loss in polycystic ovary syndrome. American Journal of Clinical Nutrition. 2006; 84: 77-87.

[17] Şanlı Ak G. Polikistik Over Sendromu Olan Kadınlarda Farklı Diyet Uygulamalarının Vücut Bileşimi ve Bazı Biyokimyasal Bulgular Üzerine Etkisi. Başkent University Health Sciences Institute Department of Nutrition and Dietetics. 2017.

[18] Yıldırım HA, Memişoğulları R. Biochemical disturbances seen in polycystic over syndrome. Konuralp Tip Derg. 2011; 3: 4248.

[19] Aydogdu A, Tasci I, Tapan S, Basaran Y, Aydogan U, Meric C, et al. High plasma level of long Pentraxin 3 is associated with insulin resistance in women with polycystic ovary syndrome. Gynecological Endocrinology. 2012; 28: 722-725.

[20] Barazzoni R, Aleksova A, Carriere C, Cattin MR, Zanetti M, Vinci $\mathrm{P}$, et al. Obesity and high waist circumference are associated with low circulating pentraxin-3 in acute coronary syndrome. Cardiovascular Diabetology. 2013; 12: 167.
[21] Osibogun O, Ogunmoroti O, Michos ED. Polycystic ovary syndrome and cardiometabolic risk: opportunities for cardiovascular disease prevention. Trends in Cardiovascular Medicine. 2020; 30: 399-404.

[22] Zweig SB, Tolentino MC, Strizhevsky M, Poretsky L. Polycystic ovary syndrome. Principles of Diabetes Mellitus. 2010; 83: 515-530.

[23] Kelly CJG, Speirs A, Gould GW, Petrie JR, Lyall H, Connell JMC. Altered vascular function in young women with polycystic ovary syndrome. Journal of Clinical Endocrinology and Metabolism. 2002; 87: 742-746.

[24] Nerkiz P, Doganer YC, Aydogan U, Akbulut H, Parlak A, Aydogdu A, et al. Serum pentraxin-3 level in patients who underwent coronary angiography and relationship with coronary atherosclerosis. Medical Principles and Practice. 2015; 24: 369375.

[25] Savchenko A, Imamura M, Ohashi R, Jiang S, Kawasaki T, Hasegawa G, et al. Expression of pentraxin 3 (PTX3) in human atherosclerotic lesions. Journal of Pathology. 2008; 215: 48-55.

[26] Singh U, Devaraj S, Vasquez-Vivar J, Jialal I. C-reactive protein decreases endothelial nitric oxide synthase activity via uncoupling. Journal of Molecular and Cellular Cardiology. 2007; 43: 780-791.

[27] Verma S, Kuliszewski MA, Li S, Szmitko PE, Zucco L, Wang $\mathrm{C}$, et al. C-reactive protein attenuates endothelial progenitor cell survival, differentiation, and function: further evidence of a mechanistic link between C-reactive protein and cardiovascular disease. Circulation. 2004; 109: 2058-2067.

[28] Lusis AJ. Atherosclerosis. Nature. 2000; 407: 233-241.

[29] Wyskida K, Franik G, Choręza P, Pohl N, Markuszewski L, Owczarek A, et al. Pentraxin 3 levels in young women with and without polycystic ovary syndrome (PCOS) in relation to the nutritional status and systemic inflammation. International Journal of Endocrinology. 2020; 2020: 1380176. 